\title{
TACKLING THE COVID-19 PANDEMIC- A REVIEW
}

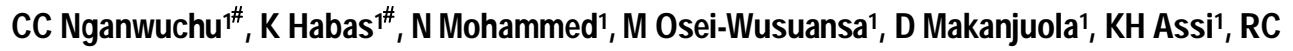 \\ Gopalan ${ }^{1}$ and MT Nasim ${ }^{1,2^{*}}$ \\ ${ }^{1}$ Translational Medicine Laboratory, School of Pharmacy and Medical Sciences, University of Bradford, \\ Bradford BD7 1DP, United Kingdom \\ ${ }^{2}$ Centre for Health, Agriculture and Socio-economic Advancements (CHASA), Lalmonirhat, Bangladesh
}

\begin{abstract}
Since December 2019, a new type of coronavirus called novel coronavirus (2019-nCoV, or COVID-19) was identified in Wuhan, China and on March 11, 2020, the World Health Organization (WHO) has declared the novel coronavirus (COVID-19) outbreak a global pandemic. With more than 101,797,158 confirmed cases, resulting in 3,451,354 deaths as of May 21, 2021, the world faces an unprecedented economic, social, and health impact. The clinical spectrum of COVID-19 has a wide range of manifestations, ranging from an asymptomatic state or mild respiratory symptoms to severe viral pneumonia and acute respiratory distress syndrome. Several diagnostic methods are currently available for detecting the coronavirus in clinical, research, and public health laboratories. Some tests detect the infection directly by detecting the viral RNA using real time reverse transcriptase polymerase chain reaction (RT-PCR) and other tests detect the infection indirectly by detecting the host antibodies. Additional techniques are using medical imaging diagnostic tools such as X-ray and computed tomography (CT). Various approaches have been employed in the development of COVID-19 therapies. Some of these approaches use drug repurposing (e.g. Remdesivir and Dexamethasone) and combinational therapy (e.g. Lopinavir/Ritonavir), whilst others aim to develop anti-viral vaccines (e.g. mRNA and antibody). Additionally, health experts integrate data sharing, provide with guidelines and advice to minimize the effects of the pandemic. These guidelines include wearing masks, avoiding direct contact with infectious people, respiratory and personal hygiene. Taken together, an extensive knowledge on transmission mechanisms, clinical spectrums, specific diagnostics methods, host-virus interactions are required to tackle this pandemic.
\end{abstract}

Key words: COVID-19, Diagnosis, SARS-CoV-2, Symptoms, Treatment

\section{Overview of COVID-19}

The global epidemic of coronavirus disease 2019 (COVID-19) has presented a major threat to public health worldwide. The pneumonia of unknown aetiology was confirmed in China in December 2019. The Chinese Centre for Disease Control and Prevention (CCDC) has identified a novel coronavirus infection as the cause of this pneumonia, and was subsequently named 2019-nCoV by WHO (Guo et al. 2020, Zhu et al. 2020a,b). In the early stages of this pneumonia, severe acute respiratory infection symptoms occurred, with some patients rapidly developing acute respiratory distress syndrome (ARDS), acute respiratory failure, and other serious complications (Guo et al. 2020). SARS-CoV-2, along with SARS-CoV and Middle East respiratory syndrome (MERS)-CoV, are classified in the genus Betacoronavirus (Zhou et al. 2020). Numerous clinical

\footnotetext{
*Author for correspondence: T.Nasim@bradford.ac.uk; \#These authors equally contributed to the work.
} 
trials are currently in progress in order to identify novel treatments. However, 2020 was a year of incredible scientific achievements. Scientists have identified a novel disease, sequenced the genome of a new virus, developed diagnostics, produced treatment protocols, and established the efficacy of drugs and vaccines in randomised controlled trials (Med 2020). Recent studies have reported that eight of the genetic variants identified, which prominently increase risk of COVID-19 mortality in patients with white British ancestry. These findings could potentially provide timely evidence for better understanding the molecular pathogenesis of COVID-19 and genetic basis of heterogeneous vulnerability, with possible impact on new therapeutic options. Thus, understanding the genetic architecture of host proteins interacting with SARS-CoV-2 could aid to identify new or repurpose existing drugs targeting those proteins (Pietzner et al. 2020). The major objective of this review is to discuss the clinical picture of SARS-CoV-2 addressing its potential causes, transmission mechanisms, diagnosis, prevention, and offer a strategic approach aiming to resolve the complicated problems of COVID-19 diseases.

\section{Clinical spectrum}

Clinical and pathological characteristics have been extensively reviewed in our earlier review article (Habas et al. 2020). The clinical spectrum of COVID-19 could range from asymptomatic infection to mild upper respiratory tract illness to severe interstitial pneumonia with respiratory failure and even death (Chen et al. 2020). The common clinical features include fever, cough, sore throat, fatigue, headache, myalgia and breathlessness. In a subset of patients, by the end of the first week the disease can progress to pneumonia, respiratory failure and death (Wang et al. 2020a,b). Less common symptoms include sore throat, confusion, headache, muscle ache, rhinorrhoea, chest pain, sputum production as well as nausea and vomiting and conjunctivitis have also been reported in some cases (Huang et al. 2020). Severe complications included ARDS, acute cardiac injury, and multiple organ failure (Huang et al. 2020). The incubation period of COVID19 was reported to be between 3 to 7 days and up to 2 weeks while the longest duration from infection to symptoms was 12.5 days (Rothan and Byrareddy 2020). The time from the initiation of COVID-19 symptoms to death varied from 6 to 41 days based on the age of the patients and the state of the patient's immune system (Li et al. 2020). Patients with severe infection had dyspnoea/hypoxemia one week after the onset. Critical patients could progress to ARDS, shock, metabolic acidosis, coagulation dysfunction, and multiple organ functional failure (Wang et al. 2020c). A recent study displayed that COVID-19 might cause various degrees damage in multiple organs include kidney and testicular tissues. Antiviral drug-induced damages to the liver were also one potential factor ( $\mathrm{Xu}$ et al. 2020). Higher mortality had been observed among older patients and patients with COVID-19 disease who have comorbidities, such as hypertension or diabetes mellitus, are more likely to develop a more severe course and progression of the disease (Table 1).

Table 1. Prevalence of underlying conditions related to high mortality in COVID-19 infection (adapted from (Hu et al. 2020).

\begin{tabular}{lc}
\hline Comorbidities & Death (\%) \\
\hline Hypertension & 15.80 \\
Cardiovascular & 11.70 \\
Diabetes & 9.40 \\
HIV and Hepatitis & 1.50 \\
Malignancy & 1.50 \\
Respiratory system (COPD and others) & 1.40 \\
Renal disease & 0.80 \\
Immunodeficiency states & 0.01 \\
\hline
\end{tabular}




\section{Diagnosis}

We earlier extensively reviewed diagnostic testing of SARS-CoV-2 (Habas et al. 2020).

\subsection{Molecular testing}

Currently, significant efforts have been made to develop the detection systems of SARS-CoV-2. The diagnosis of SARS-CoV-2 rely on the detection of the coronavirus RNA or antibodies present in the serum samples of patients (Habas et al. 2020). However, early diagnosis of COVID-19 relied on recognition of epidemiological evidence including the presence of typical clinical, laboratory, and radiographic features and the exclusion of other respiratory pathogens. The high level of SARS-CoV-2 viral load in upper and lower respiratory system have been showed in specimens of infected patients within 5-6 days of the onset of symptoms (Pan et al. 2020). For an early diagnosis of COVID-19, nasopharyngeal or oropharyngeal swabs are recommended by the WHO, however, a single nasopharyngeal swab is a method of choice for health practitioners because patients can easily provide it as well as is safe for handling. The molecular detection methods involve the analysis of nucleic acids present in the samples to identify the virus. The most reliable and commonly used laboratory detection method for the clinical diagnosis of COVID-19 is real-time RT-PCR (Corman et al. 2020).

\subsection{Serological testing}

Serological tests are one of the widely used bioanalytical methods to detect specific antibodies (IgG and IgM) in the serum that react with SARS-CoV-2 proteins. IgM is expressed earlier during an infection ( $~ 3-6$ days) and IgG is the late antibody detectable only after $\sim 8$ days (Giri et al. 2020). IgG is usually more specific for a protein antigen than IgM and affinity commonly rises with continued exposure to the viral antigen (Janeway et al. 2001).

\subsection{Laboratory examination}

A number of changes in biochemical parameters were reported in the majority of COVID-19 patients. As presented, increased levels of lactate dehydrogenase (LDH), alanine aminotransferase (ALT), aspartate aminotransferase (AST), and total serum bilirubin (TSB) and decreased levels of albumin are among the most common abnormal laboratory findings in COVID-19 patients (Pourbagheri-Sigaroodi et al. 2020). Increased levels of C-reactive protein (CRP) and high-sensitivity CRP have been reported in most of the COVID-19 patients. The highest values have been observed in the most severe cases. The elevation of other inflammatory cytokines and chemokines such as IL-8, IL-10, and TNF-a, have been found particularly in severe cases compared to mildly affected individuals (Chen et al. 2020). An increased prothrombin time (PT) together with prolonged activated partial thromboplastin time (aPTT) has also been reported in some patients (Long et al. 2020). In addition to these abnormalities, elevated D-dimers are increased and highlighted that elevation of D-dimers may effectively contribute to mirror the progression of disease toward an adverse clinical picture (Guan et al. 2020). A reduced level of overall white blood cell count (WBC) and decreased lymphocyte count are considered as the early stage of the disease (Guan et al. 2020).

\subsection{Imaging}

Imaging plays an important role in the diagnosis and follow-up of novel coronavirus infected pneumonia patients. Chest CT (Computed Tomography), a routine imaging tool for pneumonia diagnosis, is fast and relatively easy to perform. However, the $\mathrm{CT}$ is regarded as preferred imaging modality in clinically suspected cases and is valuable to monitor imaging changes within treatment (Azadbakht et al. 2020). Moreover, CT has been considered as an effective diagnostic tool for clinically suspected COVID-19 (Zhao et al. 2020). A 
recent study has been reported that CT scan findings may provide information about the disease severity (Kanne et al. 2020).

\section{Overview of the structural and functional mechanisms of SARS-CoV-2}

SARS-CoV-2 have positive RNA genome that encodes 16 non-structural proteins (NSPs) and four structural proteins which are spike (S), envelope (E), membrane (M) and nucleocapsid (N) (Schoeman and Fielding 2019). Structural proteins are mainly responsible for receptor identification in the host cell, membrane fusion, and subsequent viral entry. NSPs are crucial for replicative activities such as RNA polymerization by RNAdependent RNA polymerase (RdRp, NSP12) (Gao et al. 2020). The structural S protein forms homotrimers on the viral membrane in which each monomer is composed of two subunits; the S1 subunit comprising the receptor-binding domain $(\mathrm{RBD})$, the protein region that makes direct interaction with the host cell receptor called the angiotensin-converting enzyme 2 (ACE2) (Lu et al. 2020) and the S2 subunit comprises of the fusion peptide (FP), the heptad repeat 1 (HR1) and HR2 (Shang et al. 2020, Yan et al. 2020). Upon binding of the $S$ protein to ACE2, the host cell serine protease called transmembrane protease serine 2 (TMPRSS2) releases the S1 subunit from the $S$ protein and exposes the fusion peptide (FP) (Fig. 1) (Belouzard and Whittaker 2009). The FP initiates membrane fusion by entering the host cell membrane, prompting HR1 and HR2 to refold and form a post-fusion conformation that drives membrane fusion of the virus and target cells (White et al. 2008). RBD and ACE2 interaction is the first activity in COVID-19 cellular entry and is thus an appealing prospect for the development of COVID-19 therapy. A recent study has reported that neuropilin-1 (NRP1), which is known to bind furin-cleaved substrates, potentiates SARS-CoV-2 infectivity. NRP1 is highly expressed in the respiratory, with highest expression in endothelial and epithelial cells (Cantuti-Castelvetri et al. 2020). NRP1 could represent such an ACE2 potentiating factor by promoting the interaction of the virus with ACE2. A number of viruses use NRPs as entry factors in facilitating cell, vascular, and tissue penetration (Raaben et al. 2017), (Plein et al. 2014).

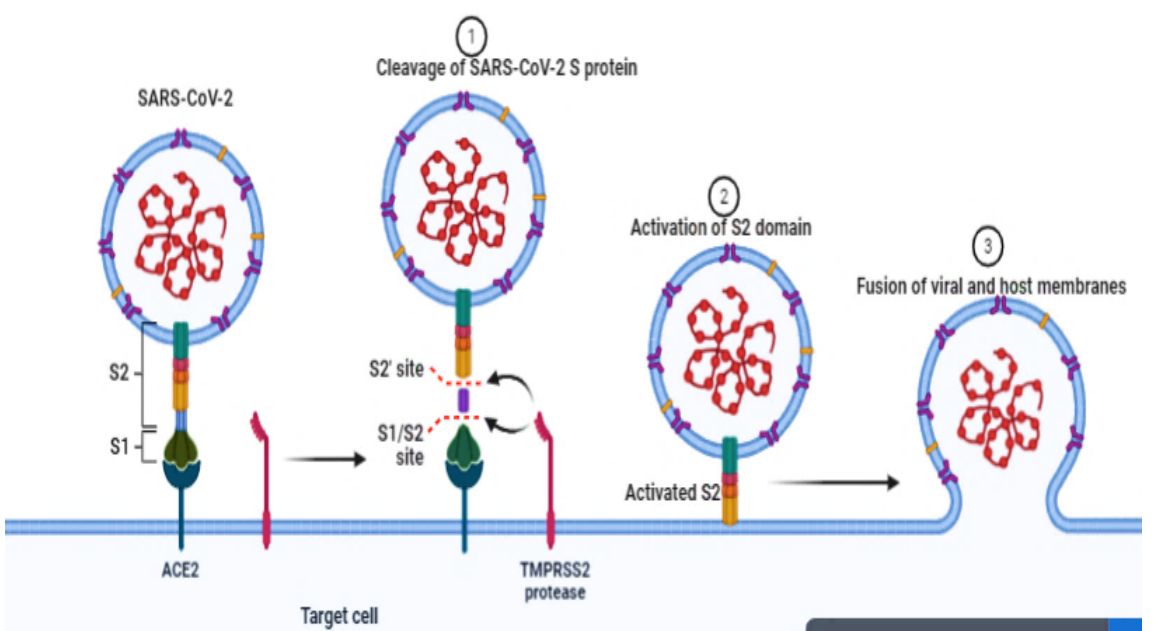

Fig. 1: The mechanisms of SARS-CoV-2 transmission and pathogenesis. The SARS-CoV-2 related coronavirus lifecycle commences via binding of the envelope Spike protein to its cognate receptor, ACE2 and TMPRSS2. 1) The cleavage of spike (S) protein to two subunits S1 and S2 sites plays key roles in the receptor recognition and cell membrane fusion process TMPRSS2. 2) The S1 subunit contains a receptor-binding domain that recognizes and binds to the host receptor ACE2. 3) While the S2 subunit mediates viral cell membrane fusion by forming a sixhelical bundle via the two-heptad repeat domain. 


\section{Therapeutic strategies for resolution of COVID-19 diseases}

More than 200 small molecule lead compounds are being considered in various stages of clinical trials. Some of the key molecules, which elicited promising results for the resolution of coronavirus diseases, have been extensively reviewed by Habas et al. (2020) and some of the recent developments are mentioned below:

\subsection{Remdesivir}

Initially screened against Ebola virus, MERS coronavirus, and other RNA viruses Remdesivir has been repurposed and approved by the FDA for the treatment of COVID-19 (Green et al. 2008, Eastman et al. 2020). Remdesivir exerts its effect by prematurely terminating the transcription of viral RNA (Wang et al. 2020). Positive results have recently been reported in a Phase III trial for Remdesivir in COVID-19 patients with moderate symptoms (Beigel et al. 2020).

\subsection{Dexamethasone}

This drug is primarily used as an anti-inflammatory or immunosuppressant agent (Price et al. 2011). Dexamethasone has recently been tested in deferent scenarios of ARDS, including viral pneumonia, and the early use of dexamethasone is safe and appears to reduce the duration of mechanical ventilation in ARDS patients. Oxford University researchers have reported progress in reducing the mortality risk of COVID-19 patients on a repurposed drug dexamethasone (NCT04381936).

\subsection{Inhibition of SARS-CoV-2 transcription and viral replication}

Lopinavir/Ritonavir: The virus SARS-CoV-2 has shown to encode specific protease which cleaves the replicase polyproteins at 11 preserved sites in order to produce the functional proteins required for viral replication (Haagmans and Osterhaus 2006). Ritonavir has been shown to be a potent CYP3A4 inhibitor and helps in drug-drug interactions as Lopinavir/Ritonavir is used in treatment of HIV. Lopinavir/Ritonavir combination has shown good efficacy as an antiviral agent in the treatment of COVID-19 because of its mechanism of action in inhibiting viral replication/transcription. Four patients with COVID-19 underwent antiviral treatment with lopinavir/ritonavir combination. Following treatment, three patients demonstrated substantial improvement in pneumonia related symptoms (Wang et al. 2020). This study showed the positive effect of Lopinavir/Ritonavir and thus has been considered as a possible treatment with phase III clinical trial being initiated for the treatment of COVID-19 patient (Yao et al. 2020).

\subsection{Inhibition of SARS-CoV-2 fusion}

Chloroquine has been widely used as an anti-malarial drug and its mechanism of action involves inhibition of viral/ cell fusion by increasing endosomal $\mathrm{pH}$ and inhibition of glycosylation whereby chloroquine interacts with sugar-modifying enzymes receptors of SARS-CoV-2 and inhibits the terminal phosphorylation of ACE2 (Savarino et al. 2006). Phase III human clinical trial has been initiated to evaluate the safety and efficacy of chloroquine in patients with COVID-19 (ChiCTR2000029609) (Wang et al. 2020c). However, the FDA has withdrawn permission for its use in that way because of adverse results in trials.

\subsection{Inhibition of SARS-CoV-2 by interferons}

Interferon $\beta$-1a: Interferon $\beta$ (IFN- $\beta$ ) is a naturally occurring protein that manipulates the antiviral reaction of the body, as the virus enters the body and fuses with cellular membranes the virus genome is released and viral replication is initiated (Zumla et al. 2016). The coronavirus has shown to be evolving and part of its mechanism is to suppress the body's production of IFN- $\beta$, helping the virus to evade the immune system and initiate viral replication. Preclinical studies with various concentrations of IFN- $\beta$ potentially inhibited the 
production of the COVID-19 in a time and drug administration-dependent manner ( $\geq 99.5 \%$ or $2.00 \log 10$ PFU/ml) (Hensley et al. 2004). The positive results have resulted in the initiation of Phase II and III trials involving IFN- $\beta$ for the treatment of COVID-19 patients (SNG001).

\section{Vaccines}

Vaccination provides an effective way of protecting people against harmful diseases by activating the immune system. All the vaccines developed or in development against COVID-19 adopt one of the following four different approaches namely mRNA vaccines (Pfizer-BioNTech and Moderna), protein subunit vaccines (Novovax), vector vaccines (Astra Zeneca) and whole virus vaccines either attenuated or inactivated (Bharat Biotech and Sinovac) (Callaway 2020b).

\section{1 mRNA- based vaccine}

Cambridge-based Moderna Inc. was the first to conduct an mRNA-based vaccine clinical trial (Phase I) (mRNA-1273, Clinical Trial Number NCT04283461, 16 March 2020). Results from the Phase I trial indicate that this vaccination has caused significant anti-virus activity (Beigel et al. 2020), which has received emergency use authorization (EUA) by the FDA.

However, on 16 March 2020, a Chinese company called CanSino announced its Phase I study of the adenovirus 5 (Ad5) vector carrying the SARS-CoV-2 S protein gene (Ad5-nCoV, NCT04341389). It was since reported substantial humoral and unique T cell responses to SARS-CoV-2 in its Phase I and II trials (Zhu et al. 2020b). Furthermore, many other therapies have since emerged, ranging from additional viral vector and nucleic acid-based designs to protein-based $\mathrm{S}$ protein subunit vaccines, as well as conventional inactivate viruses including the viral vector vaccine by Oxford/AstraZeneca Chadox1 vaccine (Gao et al. 2020).

\subsection{Protein subunit vaccine (Novovax)}

A protein subunit vaccine employs multiple dosing regimens together with adjuvants as well as numerous technical and purification processes to achieve adequate immune response (Lidder and Sonnino 2012). Nonetheless, this type of vaccine is a promising candidate against SARS-CoV-2 (Dong et al. 2020). Novavax, vaccine NVX COV2373 is a protein subunit vaccine against SARS-CoV-2 (Guebre-Xabier et al. 2020). This vaccine contains protein spikes similar to that of SARS-CoV-2 and it is formulated together with Matrix $\mathrm{M}$ an adjuvant of Novavax proprietary which improves vaccine efficacy. This lipid-saponin containing adjuvant together with NVX COV2373 stimulates high levels of neutralizing antibodies and enhances the vaccines immune response. The virus is neutralized as the serum antibodies produced, which blocks the binding between hACE2 and the viral S protein (Guebre-Xabier et al. 2020). Phase I/Phase II human clinical trials of this vaccine have demonstrated an increased in anti-S protein IgG titres with potent neutralizing activity and strong tolerability in healthy adults (18-59 years) using a two-dose regimen of $5 \mu \mathrm{g} / 25 \mu \mathrm{g}$ of Sprotein/ Matrix M respectively (Keech et al. 2020).

\subsection{Vector vaccine (Astra Zeneca)}

A COVID-19 vaccine has recently been developed by scientists from the University of Oxford and pharmaceutical company AstraZeneca, which has shown an average of $70 \%$ efficacy as evaluated in a clinical trial (Knoll and Wonodi 2020). The Oxford-AstraZeneca vaccine has been proven to be the most effective, after their second dose, 2 weeks apart (Knoll and Wonodi 2020). This vaccine is made from a common cold-causing virus isolated from the stools of chimpanzees and genetically altered so that it no 
longer replicates in cells and it cannot grow in humans from all ages and anyone with a pre-existing condition such as diabetes and hypertension. It therefore instructs human cells when injected to generate the SARSCoV-2 spike protein, which mainly targets the immune system in coronaviruses (Callaway 2020a).

\subsection{Whole virus vaccines}

Whole virus vaccine development involves the use of weakened or inactivated forms of pathogens to stimulate antibody and immune response to the disease. It is an old and established technology which has been used for the production of vaccines such as yellow fever, hepatitis A and influenza. The inactivated vaccine is known to be very safe, effective and can be administered to individuals with compromised immune systems, which includes the elderly and pregnant women (Isakova-Sivak and Rudenko 2020). It requires large quantities of the virus and a biosafety level 3 for the growth of the pathogens. Chinese companies such as Sinovac (Coronavac), Sinopharm (produced by the Wuhan institute and also the Beijing institute of biological products), Indian Bharat Biotech and the UK Valneva have produced inactivated whole virus vaccines in phase I/II clinical trials with good safety and efficacy.

\subsection{Antibody-based vaccines}

A recent study has shown that passive transfer of monoclonal antibodies against SARS-CoV-2 S protein reduced viral titers and pathology in the lungs in a mouse model of SARS-CoV-2 (Tang et al. 2020). Neutralizing antibodies derived from convalescent COVID-19 patients have been considered as potential antiviral therapeutics (Brouwer et al. 2020). Convalescent plasma use in patients has elicited a protective effect of antibodies against SARS-CoV-2 (Xia et al. 2020). Until to date eight SARS-CoV-2 neutralizing antibodies (LY-CoV555, JS016, REGN-COV2, TY027, BRII-196, BRII-198, CT-P59, and SCTA01) have entered clinical studies with many more in preclinical development (Baum et al. 2020).

\subsection{Routes of administration}

The main route of administration for the current approved COVID-19 vaccines and those under development are using intramuscular (IM) injection (Ellingson et al. 2010). However, an alternative route of administration such as intranasal (IN) could be considered should there be seasonal recurrences of COVID-19.

A study has demonstrated that the effectiveness of using intramuscular (IM) injection route of delivery for mRNA and DNA vaccines due to extended protein expression by muscle cells (Birkhoff and Marx 2009). Nevertheless, for protein-based vaccines, it was found there was a presence of high concentrations of IgA in the mucosal tissues in the lung when using IN route compared IM injection (Joo et al. 2010). There are several other advantages for considering intranasal administration compared with IM injection in term of patients' compliance. Intranasal administration is not invasive and received more conformably by certain category of patients such as children who fears injection and HIV patients who are anxious about needle stick injuries and disease transmission (Park 2020).

\section{Prevention}

Preventive measures are widely used to reduce the spread of the virus. Preventive approaches rely on isolating patients with symptoms and social distancing of asymptomatic individuals. This includes adequate steps to be taken during treatment and the delivery of health services to an infectious individual. A coordinated and multidisciplinary approach is required to provide optimal care to the patients. This includes infectious disease specialists, infection and prevention control specialists and hospital management (PeifferSmadja et al. 2020). 
The recommendations issued by the WHO and other health authorities are summarised below:

- Avoid close contact with subjects suffering from acute respiratory infections.

- Wash hands frequently, especially after contact with infected people or their environment.

- Avoid unprotected contact with farm or wild animals.

- People with symptoms of acute airway infection should keep distance, cover coughs and sneezes with disposable tissues or clothes and wash hands.

- Strengthening in emergency medicine departments, the application of strict hygiene measures for the prevention and control of infections.

- Individuals that are immunocompromised should avoid public gatherings, and no gathering of more than 20 people.

\section{Proposed strategic approaches}

To avoid possible complications, an early recognition of the disease process is essential aiming to initiate appropriate preventive and supportive treatments. Public health measures that include rapid identification, diagnosis, patient management, identification and follow-up of contacts, the prevention and control of infections in health care facilities are vital for prevention purposes. These measures are also necessary to introduce safety programmes for travellers aiming to increase public consciousness.

Multidisciplinary approach amongst public health officials and epidemiologists should be established. This will employ a variety of skills, considering the rate at which the virus is mutating. The number of mutations observed in various strains found globally may be used by genomic epidemiologists to estimate the length of existence of the virus in the environment. Identification of the possible date of introduction of the virus into a community may help estimate the number of infected persons in the community considering its contagious nature.

Finding a link between the genomic sequences of SARS-CoV-2 and its unique properties is essential in detecting if a specific mutation leads to severe forms of infection, increases rate of extreme symptoms in order to identify a better treatment and patient care.

In addition, WHO standards can be adopted as a proactive preventive step, but a one-size-fits-all approach is not enough. Countries must constantly determine which segments of community are susceptible and provide support to those at the highest risk.

\section{Conclusions}

The COVID-19 pandemic remains the greatest public health concern of this century and our knowledge to tackle and halt the outbreak is limited. Hence, substantial experiments are required to investigate the mode of transmission and pathogenicity and understand the evolutionary process from the original host to the cross-species transmission to effectively limit viral transmission. Also, revealing the complexity of the viral entry and mechanism of replication, bench to bedside translational research and reverse translational works focusing bedside to bench, may provide the foundation for the development of targeted antiviral drugs and vaccines.

\section{Competing financial interests}

The authors declare no competing financial interests. 


\section{Acknowledgement}

Research carried out at MTN Laboratories is funded by GrowMedtech, The Royal Society and University of Bradford. CN, NM, MW and DM are funded by Ph.D studentships and KH is funded by a project grant awarded to MTN.

\section{References}

Azadbakht J, Haghi-Aminjan H and Bagher F (2020). Chest CT findings of COVID-19-infected patients, are there differences between pediatric and adult patients? A systematic review. Egyptian Journal of Radiology and Nuclear Medicine, 51(1): 1-10.

Bart HL and Albert ODME (2006). Coronaviruses and their therapy. Antiviral Research, 71(2-3): 397-403.

Basant G, Shishir P, Retina S, Krisha P, Frances LS and Bhanu NB (2020). Review of analytical performance of COVID19 detection methods. Analytical and Bioanalytical Chemistry, pp. 1-14.

Baum et al. (2020). Antibody cocktail to SARS-CoV-2 spike protein prevents rapid mutational escape seen with individual antibodies. Science 369(6506):1014-1018 doi:10.1126/science.abd0831.

Beigel et al. (2020). Remdesivir for the Treatment of Covid-19 - Final Report. The New England Journal of Medicine 383(19): 1813-1826 doi:10.1056/NEJMoa2007764.

Belouzard S, Chu VC and Whittaker GR (2009). Activation of the SARS coronavirus spike protein via sequential proteolytic cleavage at two distinct sites. Proc Natl Acad Sci. USA, 106(14): 5871-5876. doi:10.1073/ pnas.0809524106.

Birkhoff M, Leitz M and Marx D (2009). Advantages of intranasal vaccination and considerations on device selection. Indian Journal of Pharmaceutical Sciences, 71(6): 729.

Brouwer et al. (2020). Potent neutralizing antibodies from COVID-19 patients define multiple targets of vulnerability. Science, 369(6504): 643-650. doi:10.1126/science.abc5902.

Callaway E (2020a). The race for coronavirus vaccines: a graphical guide. Nature, 580(7805): 576-577, doi: 10.1038/d41586-020-01221-y.

Callaway E (2020b). Why Oxford's positive COVID vaccine results are puzzling scientists. Nature, 588(7836): 16-18. doi:10.1038/d41586-020-03326-w.

Cantuti-Castelvetri L, Ravi O, Liliana DP, Minou D, Jonas F, Suvi K and Maria A (2020). Neuropilin-1 facilitates SARSCoV-2 cell entry and infectivity. Science, 370(6518): 856-860.

Chaolin H, Yeming W, Li Xingwang, Lili R, Jianping Z, Hu Yi and Xiaoying G (2020). Clinical features of patients infected with 2019 novel coronavirus in Wuhan, China. The Lancet, 395(10223): 497-506.

Cheryl K, Gary A, Iksung C, Andreana R, Patricia R, Susan N and Haixia Z (2020). Phase 1-2 trial of a SARS-CoV-2 recombinant spike protein nanoparticle vaccine. New England Journal of Medicine, 383(24): 2320-2332.

Deloria KM and Chizoba W (2020). Oxford-AstraZeneca COVID-19 vaccine efficacy. The Lancet, 397(10269): 72-74.

Dewald S and Burtram CF (2019). Coronavirus envelope protein: current knowledge. Virology Journal, 16(1): 1-22.

Gao Q, Mao BL, Wang H, Xu L, K Yang and Qin MC (2020). Development of an inactivated vaccine candidate for SARSCoV-2. Science, 369(6499): 77-81. doi:10.1126/science.abc1932.

Gao Y, Yan L, Huang Y, Liu F, Zhao Y, Cao L and Rao Z (2020). Structure of the RNA-dependent RNA polymerase from COVID-19 virus. Science, 368(6492): 779-782.doi:10.1126/science.abb7498.

Guang C, Wu Di, Guo Wei, Cao Yong, Huang Da, Wang Hongwu and Yu Haijing (2020). Clinical and immunological features of severe and moderate coronavirus disease 2019. The Journal of Clinical Investigation, 130(5). 
Guebre-Xabier, Patel M, Tian N, Zhou J-H, Maciejewski B, Lam S, Piedra K and Pedro A (2020). NVX-CoV2373 vaccine protects cynomolgus macaque upper and lower airways against SARS-CoV-2 challenge. Vaccine, 38(50): 78927896.

Habas K, Nganwuchu C, Shahzad F, Gopalan R, Haque M, Rahman S, AA Nasim and MD Talat (2020). Resolution of coronavirus disease 2019 (COVID-19). Expert Rev Anti Infect Ther, 18(12): 1201-1211. doi: 10.1080/ 14787210. 2020.1797487.

Hensley LE, Fritz LE, Jahrling PB, Karp CL, Huggins JW and Geisbert TW (2004). Interferon-beta 1a and SARS coronavirus replication. Emerg Infect Dis., 10(2): 317-319. doi:10.3201/eid1002.030482.

Hu Y, Sun J, Dai Z, Deng H, Li X, Huang Q and Xu Y (2020). Prevalence and severity of corona virus disease 2019 (COVID-19): A systematic review and meta-analysis. J Clin Virol., 127: 104371. doi:10.1016/j.jcv.2020.104371.

Hui L, Lan N, Xiaochen X, Huan L, Xiaoli Z, Wu X and Qingming (2020). D-dimer and prothrombin time are the significant indicators of severe COVID-19 and poor prognosis. BioMed Research International.

Irina I and Larisa R (2020). A promising inactivated whole-virion SARS-CoV-2 vaccine. The Lancet Infectious Diseases, 21(1): 2-3.

Janeway Jr., Charles A, Travers Paul, Mark W and Mark SJ (2001). The distribution and functions of immunoglobulin isotypes. In Immunobiology: The Immune System in Health and Disease. $5^{\text {th }}$ edition: Garland Science.

Jeffrey KP, Little Brent $\mathrm{P}$, Chung Jonathan $\mathrm{H}$, Elicker Brett M, and Ketai Loren $\mathrm{H}$ (2020). Essentials for radiologists on COVID-19: an update-radiology scientific expert panel. In: Radiological Society of North America. 296(2): 295.

Joo HM, He Y, Sundararajan A, Huan L and Sangster MY (2010). Quantitative analysis of influenza virus-specific B cell memory generated by different routes of inactivated virus vaccination. Vaccine, 28(10): 2186-2194. doi:10.1016/j.vaccine.2009.12.058.

Joshua et al. (2010). Vaccine efficacy of porcine reproductive and respiratory syndrome virus chimeras. Vaccine, 28(14): 2679-2686.

Li et al. (2020). Early Transmission Dynamics in Wuhan, China, of Novel Coronavirus-Infected Pneumonia. New England Journal of Medicine 382(13): 1199-1207 DOI: 10.1056/NEJMoa2001316.

Med and Nat (2020). COVID-19 vaccines: no time for complacency. Science, 370: 527-529.

$\mathrm{Na}$ et al. (2020). A novel coronavirus from patients with pneumonia in China, 2019. New England Journal of Medicine, 382:727-733, doi: 10.1056/nejmoa2001017e.

Nanshan C, Min Z, Xuan D, Jieming Q, Fengyun G, Yang H and Yuan W (2020). Epidemiological and clinical characteristics of 99 cases of 2019 novel coronavirus pneumonia in Wuhan, China: A descriptive study. The Lancet, 395(10223): 507-513.

Neil G, Ott Robert D, Isaacs JR and Hong F (2008). Cell-based assays to identify inhibitors of viral disease. Expert Opinion on Drug Discovery, 3(6): 671-676.

Park KS, Sun X, Aikins ME and Moon JJ (2021). Non-viral COVID-19 vaccine delivery systems. Advanced Drug Delivery Reviews, 169: 137-151. https://doi.org/10.1016/j.addr.2020.12.008.

Peiffer-Smadja et al. (2020). Challenges and issues about organizing a hospital to respond to the COVID-19 outbreak: experience from a French reference centre. Clinical Microbiology and Infection, 26(6): 669-672.

Pietzner et al. (2020). Genetic architecture of host proteins involved in SARS-CoV-2 infection. Nature Communications, 11(1): 1-14.

Plein et al. (2014). Neuropilin regulation of angiogenesis, arteriogenesis, and vascular permeability. Microcirculation, 21(4): 315-323.

Pourbagheri-Sigaroodi A, Bashash D, Fateh F and Abolghasemi H (2020). Laboratory findings in COVID-19 diagnosis and prognosis. Clin Chim Acta, 510: 475-482. 
Preetmoninder L and Andrea S (2012). Biotechnologies for the management of genetic resources for food and agriculture. In Advances in Genetics, 78: 1-167.

Price LC, Montani D, Tcherakian C, Dorfmüller P, Souza R, Gambaryan N and Howard LS (2011). Dexamethasone reverses monocrotaline-induced pulmonary arterial hypertension in rats. European Respiratory Journal, 37(4): 813822.

Raaben et al. (2017). NRP2 and CD63 are host factors for Lujo virus cell entry. Cell Host and Microbe, 22(5): 688-696.

Richard et al. (2020). Remdesivir: A Review of Its Discovery and Development Leading to Emergency Use Authorization for Treatment of COVID-19. ACS Central Science 6(5): 672-683.

Rothan HR, Byrareddy A and Siddappa N (2020). The epidemiology and pathogenesis of coronavirus disease (COVID19) outbreak. Journal of Autoimmunity, 102433.

Roujian L, Xiang Z, Juan L, Peihua N, Wu YB and Na HZ (2020). Genomic characterisation and epidemiology of 2019 novel coronavirus: implications for virus origins and receptor binding. The Lancet, 395(10224): 565-574.

Savarino et al. (2006). New insights into the antiviral effects of chloroquine. The Lancet Infectious Diseases, 6(2): 67-69.

Shang et al. (2020). Structural basis of receptor recognition by SARS-CoV-2. Nature, 581(7807): 221-224.

Song et al. (2020). Emerging 2019 novel coronavirus (2019-nCoV) pneumonia. Radiology, 295(1): 210-217.

Tang MS, Case JB, Franks CE, Chen RE, Anderson NW, Henderson JP and Farnsworth CW (2020). Association between SARS-CoV-2 neutralizing antibodies and commercial serological assays. Clin Chem., doi:10.1093/clinchem/hvaa211.

Victor et al. (2020). Diagnostic detection of Wuhan coronavirus 2019 by real-time RT-PCR. Geneva: World Health Organization, 25(3): 2000045.

Wang et al. (2020a). Clinical characteristics and therapeutic procedure for four cases with 2019 novel coronavirus pneumonia receiving combined Chinese and Western medicine treatment. Bioscience Trends, 14(1): 64-68. doi: 10.5582/bst.2020.01030.

Wang et al. (2020b). Remdesivir and chloroquine effectively inhibit the recently emerged novel coronavirus (2019-nCoV) in vitro. Cell Res, 30(3): 269-271.

Wang et al. (2020c). Unique epidemiological and clinical features of the emerging 2019 novel coronavirus pneumonia (COVID-19) implicate special control measures. Journal of Medical Virology, 92(6): 568-576.

Wei-Jie G, Zheng-Yi Hu, Yu Liang, Wen-hua Ou, Chun-Quan He, Jian-xing Hui and David SC (2020). Clinical characteristics of coronavirus disease 2019 in China. New England Journal of Medicine, 382(18): 1708-1720.

White et al. (2008). Structures and mechanisms of viral membrane fusion proteins: multiple variations on a common theme. Critical Reviews in Biochemistry and Molecular Biology, 43(3): 189-219.

Xia S, Duan K, Zhang Y, Zhao D, Zhang H, Xie Z and Yang X (2020). Effect of an Inactivated Vaccine Against SARSCoV-2 on Safety and Immunogenicity Outcomes: Interim Analysis of 2 Randomized Clinical Trials. JAMA, 324(10): 951-960. doi:10.1001/jama.2020.15543.

$\mathrm{Xu}$ et al. (2020). Pathological findings of COVID-19 associated with acute respiratory distress syndrome. The Lancet Respiratory Medicine, 8(4): 420-422.

Yan et al. (2020). Structural basis for the recognition of SARS-CoV-2 by full-length human ACE2. Science, 367(6485): 1444-1448.

Yang P, Daitao Z, Peng Y, Leo PLM and Quanyi W (2020). Viral load of SARS-CoV-2 in clinical samples. The Lancet Infectious Diseases, 20(4): 411-412.

Yan-Rong G, Qing-Dong C, Zhong-Si H, Yuan-Yang T, Shou-Deng C, Hong-Jun J and Yan Y (2020). The origin, transmission and clinical therapies on coronavirus disease 2019 (COVID-19) outbreak-an update on the status. Military Medical Research, 7(1): 1-10. 
Yao et al. (2020). A systematic review of lopinavir therapy for SARS coronavirus and MERS coronavirus-A possible reference for coronavirus disease-19 treatment option. Journal of Medical Virology, 92(6): 556-563.

Yetian D, Tong D, Yujun W, Long Z, Min Z and Fangfang Z (2020). A systematic review of SARS-CoV-2 vaccine candidates. Signal Transduction and Targeted Therapy, 5(1): 1-14.

Zhao et al. (2020). Relation between chest CT findings and clinical conditions of coronavirus disease (COVID-19) pneumonia: a multicenter study. American Journal of Roentgenology, 214(5): 1072-1077.

Zhou et al. (2020). A pneumonia outbreak associated with a new coronavirus of probable bat origin. Nature, 579(7798): 270-273.

Zhu et al. (2020a). Immunogenicity and safety of a recombinant adenovirus type-5-vectored COVID-19 vaccine in healthy adults aged 18 years or older: a randomised, double-blind, placebo-controlled, phase 2 trial. The Lancet, 396(10249): 479-488.

Zhu et al. (2020b). Safety, tolerability, and immunogenicity of a recombinant adenovirus type-5 vectored COVID-19 vaccine: a dose-escalation, open-label, non-randomised, first-in-human trial. The Lancet, 395 (10240): 1845-1854.

Zumla et al. (2016). Coronaviruses-drug discovery and therapeutic options. Nature Reviews Drug discovery, 15(5): 327347. 